Printed in Great Britain

\title{
Activation of one Tobacco Necrosis Virus by Another
}

\author{
By B. KASSANIS AND H. L. NIXON \\ Rothamsted Experimental Station, Harpenden, Hertfordshire
}

(Received 26 January 1961)

\section{SUMMARY}

Preparations of the Rothamsted culture of tobacco necrosis virus always gave two and usually three zones when centrifuged in sucrose density gradients. The top zone consisted of polyhedral particles with a sedimentation constant of $50 \mathrm{~S}$, the middle zone of larger polyhedral particles with $116 \mathrm{~S}$, and the bottom zone when present, consisted of the $50 \mathrm{~S}$ particles aggregated in groups of 12 . Neither the small particles $(50 S)$ nor their aggregates were infective when inoculated either to tobacco or French bean plants, but they became so when inoculated together with the large particles $(116 \mathrm{~S})$. The small and large particles are serologically unrelated and seem to be different viruses, one of which depends on the other for some process that allows it to multiply to detectable amounts.

The Rothamsted culture produces local lesions of different sizes in French bean. Virus isolated from single large lesions and passed through a succession of single lesions gave, when bulked in tobacco, preparations containing up to 500 large to I small particle. Evidence is given which suggests that the few small particles were acquired as contaminants when the virus was bulked in tobacco, and it seems probable that small particles would not be produced in leaves infected only with the large particles. Virus obtained from small lesions when bulked gave preparations containing particles of both sizes, with ratios of large to small particles up to 1:10. Inocula of large particles produced only large lesions, whereas mixed inocula produced large and small lesions, in proportions which depended on the ratio of the two kinds of particles in the inoculum. Two different tobacco necrosis viruses activated the small particles, but tobacco mosaic and some other viruses did not.

Particles of the two activating viruses differed in size and in their stability when negatively stained in phosphotungstate. When fixed and negatively stained they appeared to be angular, but much less so than when shadowed. Small particles tended to pack in regular arrays, and one type of packing suggested that they have a fivefold axis of symmetry.

\section{INTRODUCTION}

The group of tobacco necrosis viruses (TNV) contains several serologically unrelated viruses (Bawden, 1941) which often occur in the roots of normal looking plants and are considered to be soil borne (Smith \& Bald, 1935). The 'Rothamsted culture' of tobacco necrosis virus (RTNV) described by Bawden \& Pirie (1945, 1950) differed from other members of the TNV group and from other 'spherical' plant viruses in several respects, but particularly in its behaviour when purified. Partially purified preparations were highly infective and serologically active and always 
contained particles of at least two sizes, whereas preparations purified to the state of crystallizing consisted almost entirely of small particles, then thought to be about $17 \mathrm{~m} \mu$ in diameter, and had relatively little infectivity, though they were still serologically active. Bawden \& Pirie (1950) therefore suggested that only the large particles were infective and that the small ones, also nucleoprotein, might be derivatives from, or by-products of, the multiplication of the large particles.

Recently one of us (Kassanis, 1960) isolated from the roots of an apparently healthy tobacco plant growing in the greenhouse a TNV serologically related to RTNV but which, when examined in the electron microscope, seemed free from small particles. This suggested that the original culture of RTNV although derived from a single lesion might have been a mixture of two viruses. New attempts were therefore made to see whether the two kinds of particles could be separated by culturing from single lesions. A few of the cultures seemed to contain only large particles, but most contained both large and small particles, in different proportions in different cultures. Preparations of small particles only were obtained by centrifuging purified RTNV in sucrose density gradients. These were not infective, and leaves inoculated with them produced no lesions and yielded no small particles. Although unable to multiply by themselves, the small particles are produced when inoculated to plants also infected with the large particles.

\section{METHODS}

Single-lesion isolates were obtained from an inoculum of RTNV supplied by Mr F. C. Bawden, which was diluted so that it gave only a few lesions on tobacco leaves (Nicotiana tabacum L., White Burley var. Judy's Pride); about 1 week after inoculation selected lesions were cut from the leaves, macerated and inoculated separately to healthy tobacco plants. This process was repeated until each isolate had passed successively through six single lesions. Tobacco necrosis viruses do not become systemic, but give only necrotic lesions on the inoculated leaves of susceptible plants. The single lesion cultures had therefore to be bulked to provide enough inoculum to inoculate the large number of leaves needed to prepare virus in quantity. This was done in two stages. In the first, the extract from each single lesion was diluted in water and inoculated to as many tobacco leaves as possible. Sap from these leaves was stored in small equal samples at $-20^{\circ} \mathrm{C}$, and used to inoculate further tobacco leaves in the second stage. The sap from these leaves, again stored in equal samples at $-20^{\circ} \mathrm{C}$, was inoculated to the batches of plants used to prepare virus in quantity. In the summer, plants were covered with a black cloth for a few days before inoculation to increase their susceptibility. 'Celite' was added to all the inocula to increase the number of lesions.

The virus was purified by the methods described by Bawden \& Pirie (1957). Inoculated leaves, harvested about 1 week after inoculation, were passed through a domestic mincer and the pulp squeezed through muslin. The pulp was then extracted in a blender with a volume of water equal to the expressed sap, and squeezed again. (The pulp contained more than half as much virus as the sap.) The combined sap and extract was stored frozen at $-20^{\circ}$. When required, it was thawed and allowed to stand for $24 \mathrm{hr}$. at $20^{\circ}$ to denature normal proteins and to destroy the virus-inactivating system it contains (Bawden \& Pirie, 1957), after 
which it was centrifuged at $8000 \mathrm{~g}$ for $5 \mathrm{~min}$. The resulting brown supernatant fluid was then centrifuged at 75,000 $\mathrm{g}$ for $3 \mathrm{hr}$. The pellet was suspended in a small volume of water and left overnight at $5^{\circ}$ before centrifugation at $8000 \mathrm{~g}$ to remove insoluble material. A further cycle of high- and low-speed centrifugation gave preparations which were colourless, strongly light scattering and highly infective.

Infectivity was assayed on the primary leaves of French bean (Phaseolus vulgaris $\mathrm{L}$., var. Prince); 8 half leaves were rubbed with each inoculum.

Antisera were prepared in rabbits, by two intravenous injections of purified virus, separated by an interval of about one month. Serological tests were made either by precipitation tests in narrow tubes or by double diffusion precipitation in agar/water or agar/phosphate gels at $\mathrm{pH} 8$. The antiserum to RTNV was that used by Bawden \& Pirie (1945, 1950).

Rate zonal centrifugation in sucrose density gradients (Brakke, 1953) was used to separate the particles of different sizes using the techniques described by Harrison \& Nixon (1959). After centrifugation, the zones were removed with a micropipette which could be lowered mechanically into the gradient columns while they were being observed under vertical illumination against a black background. The samples were then dialysed against distilled water before counting the particles, or assaying infectivity or serological activity.

Particles were counted by a modification of the technique described by Nixon \& Fisher (1958). Virus samples were mixed with serum albumin, polystyrene latex suspension, and neutral sodium or potassium phosphotungstate before they were sprayed on the collodion-covered electron-microscope grids. In this way normal plant protein and microsomes could be distinguished from the small particles; also, the mounts could be examined and photographed in the electron microscope immediately, without the delay associated with the more usual shadowcasting. The actual counting was done on prints at about $\times \mathbf{7 5 , 0 0 0}$ to facilitate discrimination between large and small particles (Pl. 2, fig. 8). The electron microscope used was a Siemens Elmiskop I, and magnification calibrations were derived from a $70 \mu$ hole in a platinum aperture, measured with a light microscope. Duplicate calibration measurements were usually within $1 \%$, and we think it unlikely that our magnifications in routine work were in error by more than $3 \%$.

Particle diameters were measured with a calibrated light microscope from the original photographic plates. Usually 30 particles were measured from each plate, and images of shadowed particles were always measured at right angles to the shadowing direction, regardless of the orientation of any angular outline that was visible. All the large particles, and especially those from TNVb (see later) preparations, were heavily flattened when air dried on collodion films and then shadowed in the usual way; TNVb particles also tended to break up when mixed with neutral phosphotungstate and sprayed on the carbon-coated mounts. Both these undesirable effects could be greatly decreased by treating the virus preparations with 1-2 \% formaldehyde for a few hours, and this was done when electron micrographs suitable for measurements were needed. 


\section{RESULTS}

Separation of large and small virus particles by single-lesion isolation

Out of a total of ten single-lesion cultures prepared from the original inoculum of RTNV, four gave virus preparations which were thought at the time to contain only the large particles and six gave mixtures of large and small particles in different ratios. From these ten isolates three were selected that differed from each other in the type of lesion produced, although all reacted with antiserum to the original RTNV. The three isolates were: TNVs, containing one large to about ten small particles; TNVa and TNVb, both of which apparently contained only large particles when newly made preparations were examined with the electron microscope. However, after preparations of the last two isolates had been stored for some time, small particles were detected in them. Thus, a sample of TNVb, which initially contained fewer than one small to 500 large particles, had 3 months later about one small to 50 large particles. We think that this reflects the different stabilities of the large and small particles; when stored the preparations became enriched with the more stable small particles simply because some of the less stable large ones denatured. Small particles could also be detected in preparations derived from TNVa and TNVb by reculturing these isolates two or three times in tobacco leaves. As we shall show, this contamination might easily have come from the roots of the apparently healthy tobacco plants used for propagating and reculturing the viruses. The isolate TNVa was similar to the one found by Kassanis (1960) and referred to in the Introduction. The large particles in it were more stable than those in $\mathrm{TNVb}$, although in addition to these stable large particles, preparations of TNVa usually also contained some TNVb particles, and some small particles in about the same ratio as found in isolate $\mathrm{TNVb}$. The lesions produced on beans by TNVa appeared a day earlier, were darker in colour and spread more slowly, than those produced by $\mathrm{TNVb}$. The lesions produced by TNVa and TNVb were uniform in size, in sharp contrast to the different sizes usual on leaves inoculated with RTNV or TNVs.

Careful examination of many preparations in the electron microscope has convinced us that small particles never appeared as a result of breakdown of large ones, for images which might represent an incomplete breakdown of this sort have never been seen, although partly broken large particles were very common, particularly in unfixed negatively stained preparations of TNVb. Such breakdown seemed to be associated with the phosphotungstate, for when the particles were treated with formalin for a few hours before adding the phosphotungstate, only a few partly broken ones were seen, and the pictures resembled those obtained with TNVa (Pl. I, figs. 3, 5).

Some experiments were made to try and explain the reasons for our failure to produce pure cultures of the large particles. In one of these experiments, extracts were made from the roots of apparently healthy tobacco and of other species of plants commonly grown in the Rothamsted glasshouses. These extracts were all highly infective when inoculated to bean or tobacco leaves, and produced typical tobacco necrosis lesions. Electron microscopy showed that the extracts contained up to $1 \times 10^{11}$ small and $0.3 \times 10^{11}$ large particles/ml., or between $\frac{1}{10}$ th and $\frac{1}{10} 0$ th of the amount to be expected in inoculated leaves used for making virus preparations. 
Tobacco necrosis viruses have often been detected at Rothamsted in leaves of plants systematically infected with other viruses. This suggested that a further test might be made to see whether any of the relatively large amounts of TNV commonly found in the roots of apparently healthy plants could escape into the leaves. When sap from symptomless and apparently healthy tobacco leaves was treated as if it had contained TNV, on two out of three occasions the preparations gave an average of 2 lesions/leaf when inoculated on to bean leaves. No particles were found in these extracts with the electron microscope, but detection by electron microscopy is very much less sensitive than infectivity tests. The lower limit for detection is in the region of $10^{9}$ particles $/ \mathrm{ml}$, a concentration which gives many lesions/leaf when inoculated to beans under our conditions. TNV in small amounts has also been found in the leaves and flowers of naturally infected Primula obconica (Bawden \& Kassanis, 1947) and in the lcaves and runners of strawberry plants grown under glass (Fulton, 1952). There is thus good evidence that the roots of many apparently healthy plants contain quite high concentrations of TNV, and that significant quantities of this virus can appear in the leaves, even though these remain symptomless. Our failure to obtain and retain pure cultures of large particles is hardly surprising in these circumstances.

\section{Separation by centrifugation}

After we had failed to obtain single-lesion cultures containing only small particles, attempts were made to separate the small particles from the large by centrifuging preparations of TNVs in sucrose density gradients. After centrifuging such preparations for $2 \mathrm{hr}$. at $25,000 \mathrm{~g}$, two and sometimes three, light-scattering zones were formed. When dialysed and examined in the electron microscope, the top zone, as expected, consisted almost wholly of small particles, the middle zone of large and small particles, and the bottom zone of aggregates of small particles (to be discussed later). Separation was never quite complete. Typical ratios of small to large particles in the three zones were 100:1, 0.5:1 and 250:1 when counted in negatively stained droplet traces with the electron microscope. Because of the rapid increase of light-scattering power with increasing particle size, the bottom zone, when present, was often the most easily seen, although it contained the fewest particles. The material from the top zone was usually non-infective, so that the few large particles present in it were probably damaged and were perhaps without their nucleic acid. Damage of this kind would account for their presence in the zone of small particles each of which was only one-half to one-third of the weight of a single complete large particle, assuming the densities of the two kinds to be equal. The middle zone from the gradients was too heavily contaminated with small particles to form a useful source of the large ones, which could be obtained cleaner from preparations of TNVa and TNVb. Although it is difficult to sample one zone through another without contaminating the particles from the lower zone with some from the upper one, much better separations have been obtained in our laboratory with other viruses by using the same techniques. We think the middle zone may be contaminated by partly broken aggregates, not heavy enough to sediment with the bottom zone of complete aggregates but too heavy to remain the top zone of single small particles. Such partly broken aggregates would presumably be unstable and might break down to single particles during the subsequent dialysis. 
By measuring the depths to which the zones sedimented in the sucrose gradients and comparing these measurements with the depth to which tobacco mosaic virus sedimented in another gradient centrifuged at the same time, the sedimentation constants could be estimated. Assuming the value for tobacco mosaic virus to be $187 \mathrm{~S}$ (Lauffer, 1944), our values for the three TNV zones were $45 \mathrm{~S}, 116 \mathrm{~S}$ and $216 \mathrm{~S}$ (Kassanis \& Nixon, 1960). These figures agree well with values of $50 \mathrm{~S}$ and $116 \mathrm{~S}$ kindly obtained for us by Dr R. Markham in the analytical centrifuge with a $0 \cdot 3 \%$ virus suspension. When aggregates previously separated in a sucrose gradient were run in the analytical centrifuge, values of $49 \mathrm{~S}$ and $222 \mathrm{~S}$ were obtained on a $0.24 \%$ suspension, suggesting that some aggregates must have broken into single particles during dialysis and storage before analytical ultracentrifugation. When sap from tobacco leaves (macerated with some $\mathrm{KCN}$ ) infected with TNVs was run in the analytical ultracentrifuge, a total of six sedimenting boundaries was identified. Plate 3, fig. 14, is a typical picture; the successive peaks are, from right to left; (a) 2 peaks of normal plant protein; (b) of small particles; (c) 2 peaks of microsomes; (d) large particles. No boundary for the aggregated small particles with a sedimentation constant of $222 \mathrm{~S}$ could be found in infective sap. This makes it appear likely that the aggregates formed during purification. Our values for the sedimentation constants also agree well with those of Ogston (1942) who used preparations of RTNV made by Bawden \& Pirie (1942). Only one of the preparations examined by Ogston showed boundaries for both $116 \mathrm{~S}$ and $240 \mathrm{~S}$ components, although the preparations of TNV (apparently a mixture of RTNV with another virus) made by Pirie, Smith, Spooner \& McClement (1938) contained all three, the 116 S component in the crystalline fraction and the two others in the amorphous fraction. A possible explanation of Ogston's results is that all but one of the preparations he examined contained as their large component a high proportion of TNVb particles, which are less stable than TNVa and would therefore tend to be eliminated during the preparation of highly purified samples.

\section{The size and shape of the particles}

Negatively stained small particles looked much smaller than shadowed ones. In this respect the small particles resemble three soil-borne viruses with polyhedral particles (Harrison \& Nixon, 1960). The difference was presumably caused by the stain penetrating the outer parts of the particles, and perhaps also by slight residual flattening and consequent overestimation of the diameter of the formalin-fixed shadowed particles, which were air dried without the support of a surrounding film of phosphotungstate. By contrast, both the isolates of large particles proved to be remarkably uniform in diameter when examined in negatively stained and in shadowcast mounts (Table 1). Shadowed large TNV particles often seemed angular, and when this happened the outlines were usually hexagonal (Pl. 1, figs. 2, 4). Some particles had pointed and some flat-topped shadows, but neither the angular outlines nor the shadow shapes appear consistently enough to permit any useful deductions to be made about the exact shape of the large TNV particles, except the very general one that they are almost certainly polyhedral. This impression is supported by the somewhat angular appearance of the large particles in negatively stained preparations ( $\mathrm{Pl}$. 1, figs. 1 and 3). Small particles also looked angular when fixed with formalin before shadowing, and tended to pack into regular arrays in 
negatively stained mounts (PI. 2, fig. 7). Much of the apparent angularity in negatively stained preparations is an illusion caused by regular packing.

By studying the various ways in which the small particles packed some deductions can be made about the symmetry of each individual particle. We are indebted to Mr G. Brown of the Pedology Department at Rothamsted for the interesting suggestion that the appearance of an angle of $72^{\circ}\left(360^{\circ} / 5\right)$ between the lines of particles in one type of packing (Pl. 2, fig. 7) indicates that each of the small particles may have a fivefold axis of symmetry. The presence of fivefold axes of symmetry is one of the features of the (532) symmetry that has recently been demonstrated by $\mathbf{X}$-ray diffraction and electron microscopy in some other virus particles. This evidence for fivefold symmetry in the small TNV particle suggests that it may possibly have similar symmetry.

Table 1. The size of tobacco necrosis virus particles (TNV) estimated from electron micrographs made by two different techniques

$\begin{array}{lc}\text { Material } & \text { Diameter }(\mathrm{m} \mu) \\ \text { TNVa } & \\ \quad \text { Stained } & \mathbf{2 9 \cdot 8 8} \pm \mathbf{0 \cdot 6 0} \\ \text { Shadowed } & \mathbf{2 9 \cdot 6 \theta} \pm \mathbf{0 \cdot 3 6} \\ \text { TNVb } & \\ \text { Stained } & \mathbf{2 5 \cdot 0 5} \pm \mathbf{0 \cdot 4 4} \\ \quad \text { Shadowed } & \mathbf{2 6 \cdot 8 3} \pm \mathbf{0 \cdot 4 1} \\ \text { Small particle } & \\ \quad \text { Stained } & \mathbf{1 4 \cdot 2 8} \pm \mathbf{0 \cdot 1 9} \\ \text { Shadowed } & \mathbf{2 1 \cdot 2 1} \pm \mathbf{0 . 6 0}\end{array}$

The aggregates which formed the bottom zone after centrifugation in sucrose were visible only in negatively stained mounts, and even in these they sometimes collapsed into groups of small particles (Pl. 2, fig. 8). Aggregates occurred in three orientations, and the images can all be accounted for by assuming that each aggregate consisted of twelve small particles, arranged in two rings of five placed back to back, with an additional particle placed at the centre of each ring (Pl. 1, fig. 6). The most common number of particles found in the groups thought to represent collapsed aggregates was twelve, which agrees well with the conclusion about the structure of the aggregates reached from micrographs of the aggregates themselves. Small particles taken from broken aggregates were indistinguishable in size and appearance from those in preparations of unaggregated small particles taken from the top zone after centrifugation in sucrose density gradients. Neither aggregates nor groups thought to represent collapsed aggregates were found in shadowed mounts, even when aggregates were common in negatively stained mounts made from the same virus preparations. Presumably the aggregates were destroyed by drying them without a supporting film of phosphotungstate and the resulting small particles were scattered over the mount in the remaining liquid film, so that no discrete groups could be recognized. It is interesting to note that the aggregate structure also has the (532) symmetry already discussed. 


\section{Infectivity tests}

When the zones in the density gradient tubes were clearly separated after centrifugation and the sampling carefully done, only material from the middle zone was infective and produced lesions when inoculated to bean or tobacco leaves. Leaves inoculated with preparations of small particles from the top zone not only failed to produce lesions, but no virus-like particles could be detected, either by serology or electron microscopy, in clarified sap from the leaves or after the sap had been subjected to the procedures used to purify and concentrate the virus. In contrast, extracts from leaves inoculated with a mixture of small particles from the top zone of a sucrose density gradient and large particles from $\mathrm{TNVa}$ or $\mathrm{TNVb}$ preparations, contained small and large particles in roughly equal numbers (Table 2). Similarly, aggregates from the bottom zone did nothing alone, but multiplied when inoculated together with TNVa or TNVb. Old preparations of TNVa, substantially free from the less stable $\mathrm{TNVb}$ particles, were as efficient as fresh preparations in aiding the multiplication of small particles. Both the large particles therefore

Table 2. The ratio of large to small particles in virus preparations purified from tobacco plants inoculated with large particles of tobacco necrosis (TNV) alone or in mixtures with the small particles

\begin{tabular}{cccccc} 
& \multicolumn{5}{c}{ Ratio:large/small } \\
Virus inoculum* & Expt....1 & 2 & 3 & 4 & 5 \\
TNVb (large) & - & - & 20 & 83 & - \\
TNVa (large) & 12.5 & $\mathbf{2 0 \cdot 6}$ & - & - & 13 \\
Large† + small & $\mathbf{1 . 3}$ & 0.9 & $\mathbf{2 . 2}$ & $\mathbf{0 . 2}$ & $\mathbf{0 . 7}$ \\
Small & $\mathbf{N o}$ virus detected. & & &
\end{tabular}

* The leaves were inoculated with the aid of 'Celite'; the concentrations of inoculum in mg./1. for the large and small particles were, respectively: Exp. 3, 4 and 100; Exp. 4, 40 and 40; Exp. 5, 40 and 100.

$\dagger$ The large particles used in Exps. 1, 2 and 5 were TNVa and in Exps. 3 and 4 TNVb.

activated the small ones. The ratio of the two kinds of particle in the inoculum was not the only factor that determined the ratio in the product, for when young and old tobacco leaves were inoculated with the same mixed inoculum and harvested separately, the ratio of small to large particles was 50:1 in the virus preparation made from young leaves and 16:1 in a similar preparation made from old leaves, suggesting that young leaves were relatively more susceptible than old leaves to the small particle. From these results we conclude that, whereas the large particles can infect and multiply unaided, the small ones cannot, and multiply only when they are in leaves together with the large. If this be so, then it should also be possible to obtain pure isolates of the large particles which can be cultured repeatedly without becoming contaminated with small ones. This we were unable to do, for, as already described, many healthy looking tobacco plants contain TNV.

The results in Table 2 are from experiments in which the large and small particles were inoculated to tobacco plants simultaneously. The small particles were also activated when leaves were inoculated with the two kinds of particles at different times. For example the ratios of large to small virus particles in virus preparations 
obtained from tobacco leaves inoculated with the same preparation of the two particles but at different times were: $\mathbf{0 . 2}$ when the leaves were inoculated with both particles simultaneously; 1.2 when the small particles were inoculated 2 days before the large $(\mathrm{TNVb}) ; \mathbf{5 . 9}$ when the large particles were inoculated 2 days before the small; and 23 when inoculated with the large particles only. Most small particles were produced when a mixed inoculum was used, and more when the small particles were inoculated before the large than afterwards. No other tobacco necrosis viruses have been tested, but tobacco mosaic, lucerne mosaic, carnation ringspot, and tomato bushy stunt viruses all failed to make the small particles multiply.

Preparations of TNVa disrupted by phenol are highly infective (Kassanis, 1960). Phenol-disrupted preparations of the small particles, like the whole small particles, did not multiply in bean or tobacco plants. When, however, such preparations were mixed with TNVa or TNVb, either intact or phenol-disrupted, the small particles multiplied. In one such experiment the ratio of large to small particles was $2 \cdot 4$ in virus preparations made from tobacco plants inoculated with a mixture of phenoldisrupted small particles and TNVa, and 40 in preparations from plants inoculated with disrupted TNVa alone.

\section{Inhibition of lesion formation}

Tobacco and bean leaves inoculated with TNVs produced a mixture of small and large lesions, whereas TNVa, TNVb gave only large-size lesions (Pl. 3, figs. 11, 12). When inocula of $\mathrm{TNV}$ a or $\mathrm{TNVb}$ were mixed with different concentrations of small particles, the number of small lesions increased with increasing number of small particles in the inoculum (Table 3). In the experiment in Table 3 the total number of lesions remained about the same with all concentrations of small particles, but when the concentration of small particles in the inoculum increased much above $5 \mathrm{mg}$./l. the total number of lesions decreased (Kassanis \& Nixon, 1960).

Table 3. The infectivity of tobacco necrosis virus $(T N V b)$ in the presence of different amounts of small particles

$\begin{array}{ccc}\begin{array}{c}\text { TNVb } \\ \text { Concentration of } \\ \begin{array}{c}\text { small particles } \\ \text { (mg./l.) }\end{array}\end{array} & \begin{array}{c}\text { Number of lesions on } 8 \text { half } \\ \text { leaves of French beans }\end{array} \\ 5 & \text { Large lesions } & \text { Small lesions } \\ 1.25 & 20 & 274 \\ 0.31 & 44 & 199 \\ 0.08 & 157 & 95 \\ 0 & 151 & 33 \\ & 217 & 0\end{array}$

Freshly prepared TNVa or TNVb produced only large lesions, and behaved as if they contained only large particles, presumably because the fresh preparations had to be diluted considerably to give countable lesions, and this dilution lowered the concentration of small particles in the inoculum to a concentration below that needed to infect. Stored preparations, however, had to be diluted less to give the same number of lesions, and these gave some small lesions. 


\section{Serological tests}

From plants inoculated with small particles only, nothing could be isolated that precipitated specifically with antiserum to TNVs (mixed particles), whereas virus prepared from plants inoculated with the small and large particles gave two lines in gel diffusion tests, showing that the two particles were serologically unrelated. Plate 2, figs. 9 and 10, show the results of two such tests. In both tests the three upper cups contained, from left to right, virus prepared from tobacco leaves inoculated with $(a)$ small particles alone; $(b)$ large particles alone; $(c)$ the mixture. For Pl. 2, fig. 9, antiserum to TNVs was placed in the centre cup and, for Pl. 2, fig. 10, the antiserum was to large particles only. Fig. 10 shows only one precipitation line, and fig. 9 shows two lines near the top right cup which contains the virus produced from mixed inoculum. Similar results were obtained with TNVa and TNVb.

\section{Table 4. Dilution end points of antisera tested against homologous and heterologous viruses}

$\begin{array}{llll}\text { Antiserum } & \overbrace{\begin{array}{c}\text { Small } \\ \text { particles } \\ \text { (Top zone) } \\ \text { End-point dilution }\end{array}}^{\text {TNVa }} & \text { TNVb } \\ \text { TNVa } & \overbrace{0(1 / 5) *}^{1 / 160} & - \\ \text { TNVb } & 0(1 / 5) & - & 1 / 80 \\ \text { TNVs (mixture) } & 1 / 160 & 1 / 80 & 1 / 80 \\ \begin{array}{c}\text { TNVs (mixture) } \\ \text { absorbed with TNVa }\end{array} & 1 / 160 & 0(1 / 20) & - \\ \begin{array}{c}\text { TNVs (mixture) } \\ \text { absorbed with TNVb }\end{array} & 1 / 160 & - & 0(1 / 20) \\ \end{array}$

* The figures in parentheses show the highest concentration at which the antisera were tested.

The absence of any detectable serological relationship between the large and small particles was also demonstrated in the three lower cups in Pl. 2, fig. 9, which contained mixtures of large and small particles in different ratios, for the two precipitation lines cross. In Pl. 2, fig. 10, the same set of mixtures gave only one line when tested against an antiserum to large particles only. Precipitation tests in tubes also failed to show any common antigens between the small particles and either TNVa or TNVb (Table 4). Antiserum to either of the two large particles did not precipitate at dilutions greater than $1 / 5$ with the small particles obtained from the top zone of sucrose density gradient columns, but each precipitated against preparations of the corresponding homologous particle. Also, the antiserum to TNVs (mixed particles), when absorbed with either of the two large particles TNVa or TNVb, still precipitated against preparations of small particles to the same dilution as it did when unabsorbed. So far we have not established a serological relationship between $\mathrm{TNVa}$ and $\mathrm{TNVb}$. 


\section{DISCUSSION}

The small particles evidently multiplied detectably only in the presence of the large particles; but our results do not show with the same degree of certainty that the large particles could multiply without producing any small ones. A reasonable explanation for the preparations of TNVa and TNVb containing a few small particles is that they became contaminated when propagated in tobacco plants, for we have shown how easily leaves can become infected from the heavily infected roots. However, there are other possibilities. First, although some single lesion isolates consist only of large particles, small particles might appear as mutants, for the possibility that the two kinds of particles are remotely related cannot be excluded. The fact that they are of different sizes does not in itself eliminate the possibility of a distant relationship between them, for such relationships have recently been shown between a number of rod-shaped viruses with different normal lengths (Bercks, 1960; Wetter, Quantz \& Brandes, 1959). Alternatively, the association between large and small particles may be so close that it is not broken by the methods we have used. On this view repeated single-lesion isolation might merely alter the ratio in favour of the large particles, and the ratio would then gradually return to its previous value when the culture was propagated without continuous selection. This second possibility casts some doubt upon the ability of the large particles to function without the small; but until the contrary is proved we think it reasonable to assume that the large particles neither need the small ones nor directly produce them.

None of the properties of the small particles so far investigated suggests that they should not be called virus particles, for in some circumstances they infect and multiply. Like the large particles, they consist of nucleoprotein and, in common with other tobacco necrosis viruses, they contain from 1.7 to $2.0 \%$ phosphorus (Bawden $\&$ Pirie, 1942). The difference in size means that each small particle has only about one-third to one-half of the volume of a large one and, assuming that the densities of the two kinds to be the same, each small particle will contain a correspondingly small amount of nucleic acid. The particles are smaller than other known spherical viruses and their inability to multiply on their own may reflect an inadequacy of nucleic acid and the need to 'borrow' some missing part from the large particles.

Finally the possibility must be considered that the small particles do multiply, but without causing any symptoms and without reaching concentrations high enough to be detected even by infectivity tests. In the presence of the large particles, the concentration of the small ones might rise to the concentration found in normal mixed cultures. There are other examples of one virus increasing the concentration of another (Rochow \& Ross, 1955; Kassanis, 1961), but with these the increase is small compared with the amount reached by the one virus inoculated alone. If the small particles do multiply on their own, perhaps in the cells they enter at the time of inoculation, they do not reach even 1/1000th the concentration they reach in mixed cultures with the large particles, for if they did they would have been detected with the electron microscope. Movement from cell to cell, however, might be the ability conferred on the small particles by the large; but until there is evidence of multiplication in the initially infected cells, there is no good reason for assuming this, rather than any other, to be the critical stage aided by the large particles. 
Further evidence, about possible relations between large and small particles and about the continued ability of the large virus to multiply without producing any small particles, is unlikely to be obtained until the viruses can be propagated in conditions free from the risk of contamination by tobacco necrosis viruses present in the roots and leaves of apparently healthy tobacco plants.

We are indebted to $\mathrm{Mr} \mathrm{R}$. D. Woods for his able assistance with the electron microscopy.

\section{REFERENCES}

BAwDEN, F. C. (1941). The serological reactions of viruses causing tobacco necrosis. Brit. J. exp. Path. 22, 59.

Bawden, F. C. \& Kassanis, B. (1947). Primula obconica, a carrier of tobacco necrosis viruses. Ann. appl. Biol. 34, 127.

Bawden, F. C. \& Pirie, N. W. (1942). A preliminary description of preparations of some of the viruses causing tobacco necrosis. Brit. J. exp. Path. 23, 314.

Bawden, F. C. \& Pirie, N. W. (1945). Further studies on the purification and properties of a virus causing tobacco necrosis. Brit. J. exp. Path. 26, $27 \%$.

Bawden, F. C. \& Pirie, N. W. (1950). Some factors affecting the activation of virus preparations made from tobacco leaves infected with a tobacco necrosis virus. J. gen. Microbiol. 4, 464.

Bawden, F. C. \& Pirie, N. W. (1957). A virus-inactivating system from tobacco leaves. J. gen. Microbiol. 16, 696.

BerCKs, R. (1960). Serological relationships between beet mosaic virus, potato virus $Y$ and bean yellow mosaic virus. Virology, $12,311$.

BrakKe, M. K. (1953). Zonal separation by density gradient centrifugation. Arch. Biochim. Biophys. 45, 275.

Fulton, J. P. (1952). A tobacco necrosis virus associated with strawberry plants. Plant Dis. Reptr, 36, 313.

Harrison, B. D. \& Nixon, H. L. (1959). Separation and properties of particles of tobacco rattle virus with different lengths. J. gen. Microbiol. $21,569$.

Harrison, B. D. \& Nixon, H. L. (1960). Purification and electron microscopy of three soil-borne plant viruses. Virology, 12, 104.

Kassanis, B. (1960). Comparison of the early stages of infection by intact and phenoldisrupted tobacco necrosis virus. Virology, 10, 353.

Kassanis, B. (1961). The transmission of potato aucuba mosaic virus by aphids from plants also infected by potato viruses $A$ or Y. Virology, 13, 93.

Kassanis, B. \& Nixon, H. L. (1960). Activation of one plant virus by another. Nature, Lond. 187, 713.

LAUFFER, M. A. (1944). The influence of concentration upon the sedimentation rate of tobacco mosaic virus. J. Amer. Chem. Soc. 66, 1195.

Nixon, H. L. \& Fisher, H. L. (1958). An improved spray droplet technique for quantitative electron microscopy. Brit. J. appl. Phys. 9, 68.

Ogston, A. G. (1942). Addendum: Examination in the ultracentrifuge. Brit. J. exp. Path. 23, 328.

Pirme, N. W., Smith, K. M., Spooner, E. T. C. \& McClement, W. D. (1938). Purified preparations of tobacco necrosis virus (Nicotiana virus II). Parasitology, 30, 543.

Rochow, W. F. \& Ross, A. F. (1955). Virus multiplication in plants doubly infected with potato viruses $X$ and $Y$. Virology, $1,10$.

SmrTH, K. M. \& BALD, J. G. (1935). A description of a necrotic virus disease affecting tobacco and other plants. Parasitology, 27, 231.

WeTter, C., QuANTz, L. \& Brandes, J. (1959). Verwandtschaft zwischen dem Stauchevirus der Erbse und dem Rotkleeadernmosaik-Virus(red clover vein mosaic virus). Phytopath. $Z$. 35, 201. 

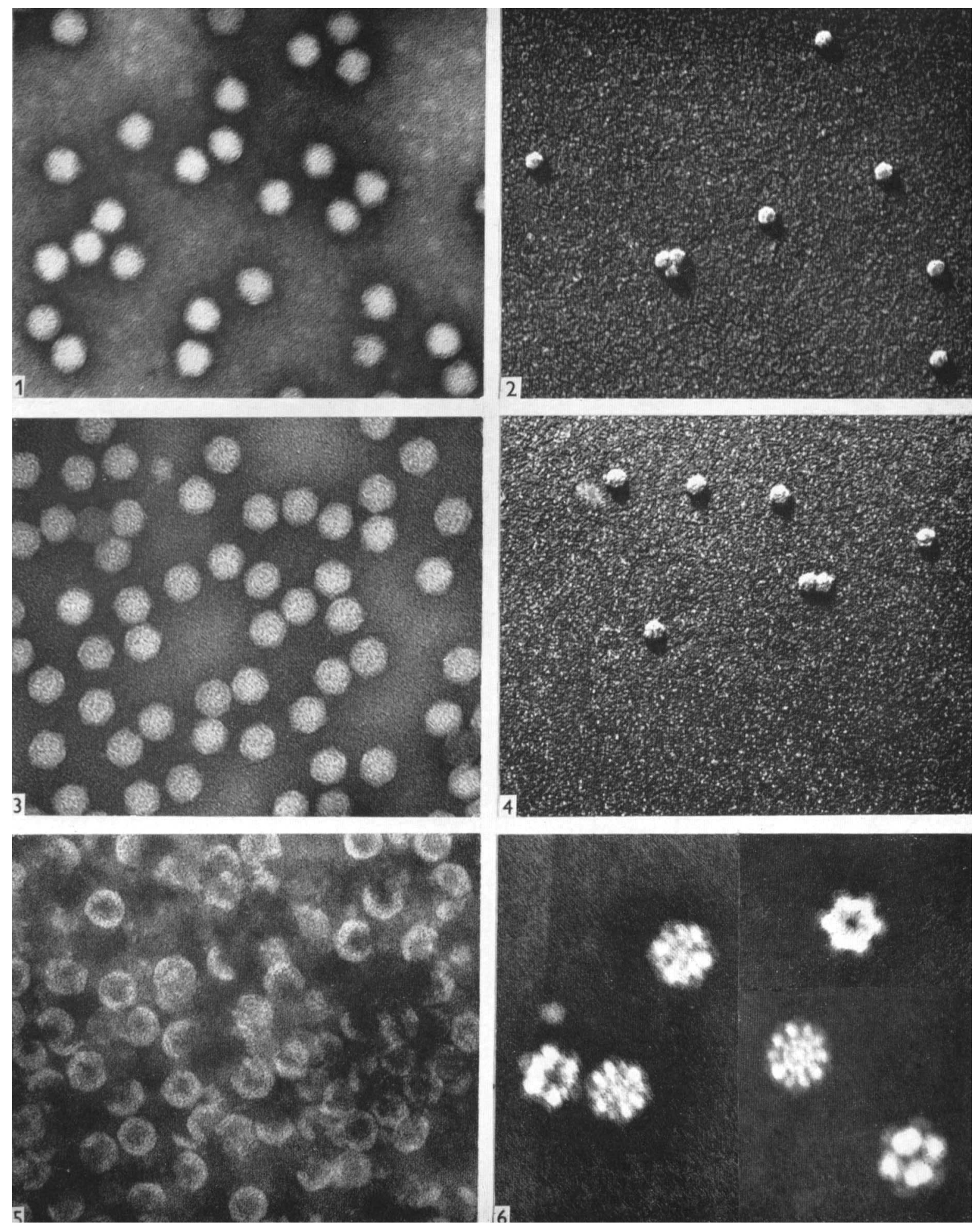

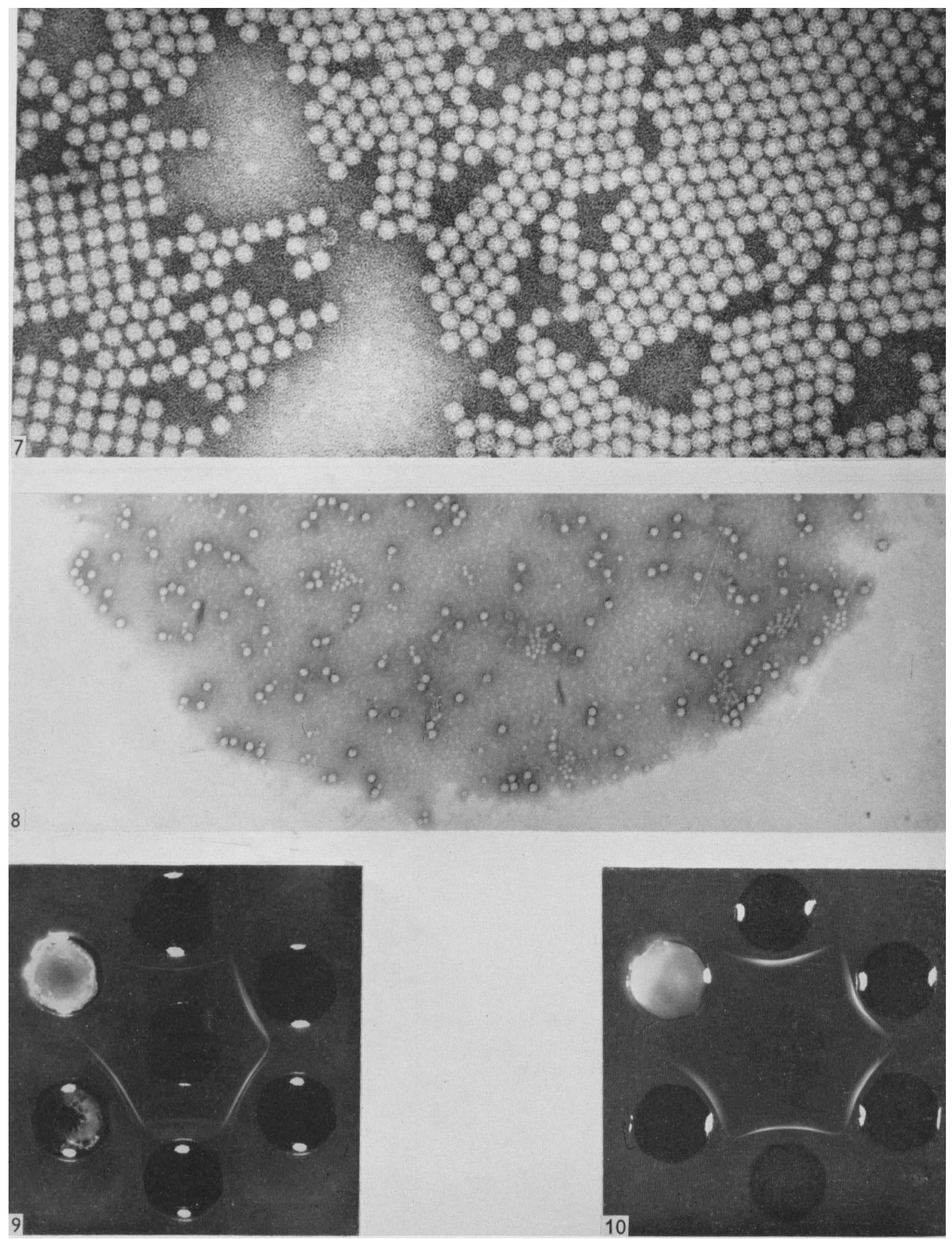

B. KASSANIS AND H. L. NIXON 


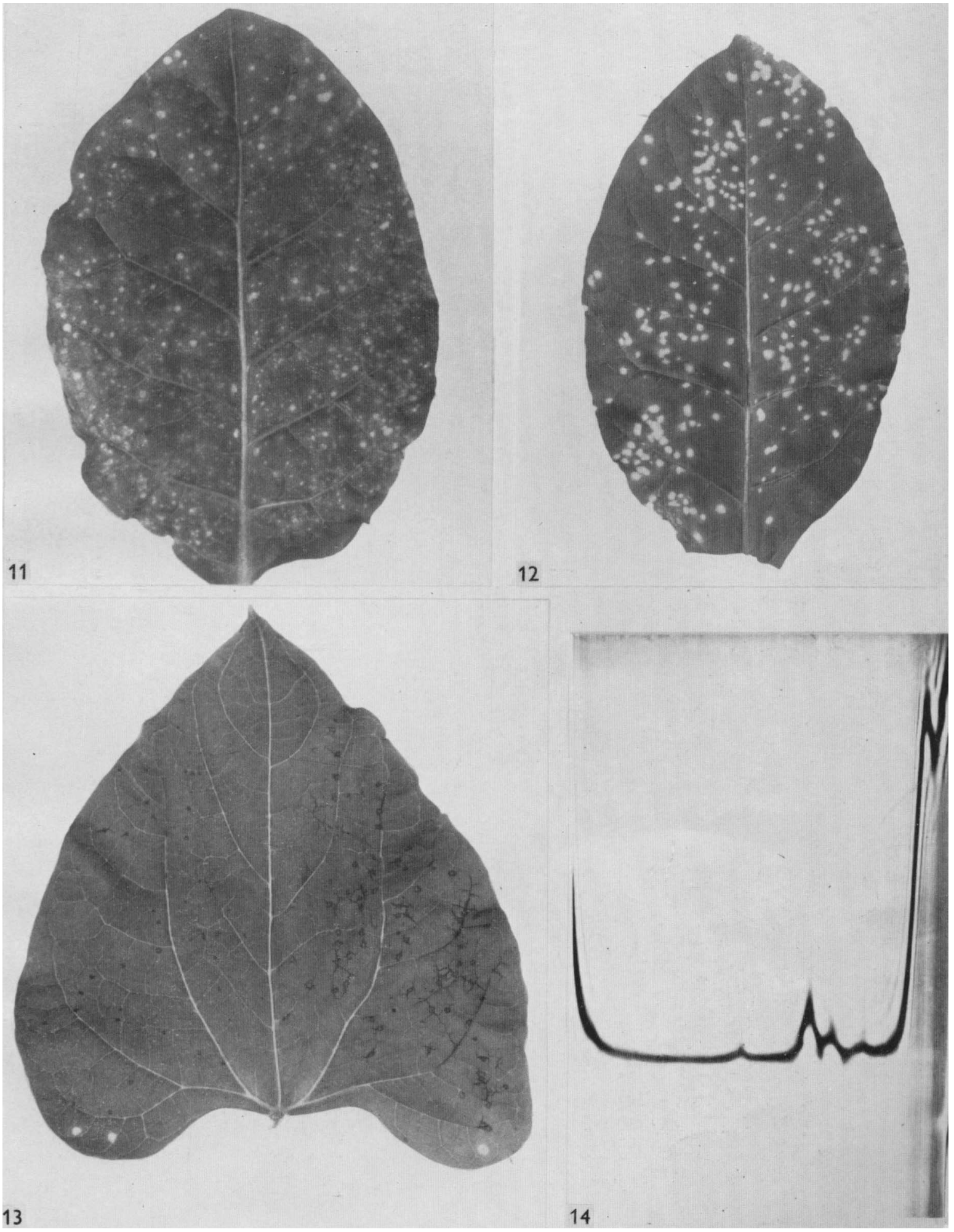

B. KASSANIS AND H. L. NIXON 



\section{EXPLANATION OF PLATES}

\section{Plate 1}

Fig. 1. TNVa particles fixed with formalin and mounted in phosphotungstate. $\times 240,000$ approx. Fig. 2. TNVa particles fixed with formalin and shadowed with platinum-iridium alloy. $\times 108,000$. Fig. 3. TNVb particles fixed with formalin and mounted in phosphotungstate. $\times 240,000$ approx. Fig. 4. TNVb particles fixed with formalin and shadowed with platinum-iridium alloy. $\times 108,000$. Fig. 5. TNVb particles mounted in phosphotungstate without fixation. $\times 240,000$ approx.

Fig. 6. Aggregates, each consisting of 12 small particles, from the bottom zone of a preparation of TNVs after centrifuging in a sucrose density gradient. Mounted in phosphotungstate. $\times 240,000$ approx.

\section{Prate 2}

Fig. 7. Small particles from the top zone of a preparation of TNVs after centrifuging in a sucrose density gradient, mounted in phosphotungstate. Three different types of packing can be seen. Many particles on the right of the picture are packed in rows inclined at $72^{\circ}$, suggesting that they are oriented with a fivefold axis emerging at the centre of each particle. $\times 240,000$ approx.

Fig. 8. Part of a droplet trace made by a virus preparation (TNVa) from plants inoculated with large and small particles, mixed with phosphotungstate, showing large and small particles. Some of the large particles have been penetrated by the phosphotungstate and resemble those shown in Fig. 5. $\times 74,000$.

Fig. 9. Gel diffusion test in which the 3 upper cups contained from left to right: $(a)$ virus preparation from plants inoculated with small particles; $(b)$ large; and $(c)$ large and small particles. The three lower cups contained virus preparations with different ratios of large and small particles, the central cup having more small particles than large and those on each side of it more large than small. The cup in the centre contained antiserum to both large and small particles.

Fig. 10. Gel diffusion test as in Fig. 3 but with antiserum to large particles in the centre.

\section{Plate 3}

Figs. 11, 12. Tobacco leaves inoculated with two kinds of particles; in fig. 11 the inoculum was a mixture of large and small particles, in fig. 12 the inoculum was large particles only.

Fig. 13. Bean leaf inoculated with two kinds of particles; the left half with large and small particles, the right with large particles only.

Fig. 14. Sedimentation pattern of sap from leaves infected with TNVs. The boundaries are, from right to left: two large peaks of normal plant protein, a very small peak with $S=43$ (small virus particles), two larger peaks with $S=58$ and 69 (microsomes), and a very small peak with $S=108$ (large virus particles). 